\title{
Comparison and Problems of Measured Values of LH, FSH, and PRL among Measurement Systems
}

\author{
TAKESHI IWASA, TOSHIYA MATSUZAKI, NAOKO TANAKA, MASAHIRO MINAKUCHI, FUMI SHIMIZU, \\ RIE OGATA, MACHIKO KIYOKAWA, MASAHIKO MAEGAWA, TOSHIYUKI YASUI, AKIRA KUWAHARA \\ AND MINORU IRAHARA
}

Department of Obstetrics and Gynecology, University of Tokushima School of Medicine, 3-18-15, Tokushima 770-8503, Japan

\begin{abstract}
Several measurement systems are used for LH, FSH, and PRL in Japan, but the comparison of the measured values is difficult in some cases. In this study, we compared the measured values of 3 types of measurement systems widely used in Japan, SPAC-S, ARCHITECT, and Centaur, in females with a normal menstrual cycle and with various ovulatory disorders. Variant LH was discriminated by the criterion, SPAC-S LH/ARCHITECT LH ratio $<0.5$. Excluding the variant LH, the correlation of the measured LH values among the measurement systems was high, 0.94-0.99, and the value measured by ARCHITECT LH and Centaur LH was slightly higher than that measured by SPAC-S LH. As for FSH, the correlation was also high, 0.94-1.00, and the value measured by ARCHITECT FSH and Centaur FSH was slightly lower than that measured by SPAC-S FSH. The important judgment criterion of polycystic ovary syndrome (PCOS), a high level of LH relative to FSH, has been set to 1.0 or higher LH/FSH using SPAC-S. When the regression line of the LH/FSH ratio among the measurement systems was investigated, it can be said that 1.25 or higher by ARCHITECT and 1.40 or higher by Centaur are appropriate for the judgment criteria of a high level of LH relative to FSH. As for PRL, the correlation was high, 0.96-0.99, but the value showed large differences among the systems. For the diagnosis of hyperprolactinemia, the higher limit of the normal range may be $15 \mathrm{ng} / \mathrm{mL}$ for SPAC-S, $30 \mathrm{ng} / \mathrm{mL}$ for ARCHITECT, and $24 \mathrm{ng} / \mathrm{mL}$ for Centaur.
\end{abstract}

Key words: SPAC-S, ARCHITECT, Centaur, Variant LH, PCOS

(Endocrine Journal 53: 101-109, 2006)

SEVERAL measurement systems of LH, FSH, and PRL are currently used in Japan, but the comparison of the measured values among the measurement systems is difficult because the standards are not identical. In this study, we compared the values measured using 3 measurement systems widely used in Japan, SPAC-S (Daiichi Laboratories, Ltd., Tokyo, Japan), ARCHITECT (Abbott Japan Inc., Tokyo, Japan), and Centaur (Bayer Medical Co., Ltd., Tokyo, Japan), using samples from women with normal menstrual cycles, patients with various ovulatory disorders, postmenopausal women, and pregnant women.

Received: July 4, 2005

Accepted: November 4, 2005

Correspondence to: Takeshi IWASA, M.D., Department of Obstetrics and Gynecology, University of Tokushima School of Medicine, 3-18-15, Tokushima 770-8503, Japan

\section{Materials and Methods}

Subjects

Thirty-six women with normal menstrual cycles (273 samples), 45 patients with various ovulatory disorders (107 samples), 11 postmenopausal women (11 samples), and 28 pregnant women ( 28 samples) were enrolled in this study. Written informed consent was obtained from all patients. Anovulatory cycle was diagnosed by cyclic genital bleeding with luteal phase defect in basal body temperature. Amenorrhea was defined as loss of menstrual bleeding for more than 3 months. Amenorrheic patients who had genital bleeding after oral progestogen administration were diagnosed as hypothalamic normoestrogenic amenorrhea, and amenorrheic patients who did not have such withdrawal bleeding were diagnosed as hypothalamic 
hypoestrogenic amenorrhea. Amenorrheic patients with hyper serum FSH concentration more than $30 \mathrm{mIU} / \mathrm{mL}$ were diagnosed as ovarian amenorrhea. PCOS was diagnosed using the criteria of Japanese Society of Obstetrics and Gynecology. It consisted of the association of all of the following 3 factors and the exclusion of other endocrine disorders: 1 . clinical findings of amenorrhea or oligomenorrhea and presence of chronic anovulation, 2. higher LH concentrations (LH/FSH $>1$ as measured by SPAC-S [1]), 3. presence of polycystic ovaries. Luteal insufficiency was diagnosed by short luteal phase less than 11 days and/or serum progesterone level less than $10 \mathrm{ng} / \mathrm{mL}$ in mid luteal phase measured by AIA (TOSOH Bioscience, Inc. Tokyo, Japan). Hyperprolactinemia was diagnosed by serum PRL level more than $15 \mathrm{ng} / \mathrm{mL}$ measured using SPAC-S PRL.

\section{Methods}

The measurements were performed using 3 measurement systems SPAC-S (immunoradiometric assay (IRMA)), and ARCHITECT and Centaur (chemiluminescence immunoassay (CLIA)). Regarding the standards, ARCHITECT LH and Centaur LH use an identical LH standard, SPAC-S FSH and ARCHITECT FSH use an identical FSH standard, and ARCHITECT PRL and Centaur PRL use an identical PRL standard (Table 1). The main analytical characteristics of these immunoassays are summarized in Table 2-4. In all samples, LH, FSH, and PRL concentrations were measured by these 3 measurement systems, and correlations among the systems were investigated. The base values of LH, FSH, and PRL in women with normal menstrual cycles, circadian and intra-menstrual cycle variations, variations in the gestational periods, and values in patients with various ovulation disorders were investigated. We defined the criteria of variant LH as SPAC-S LH/ARCHITECT LH ratio less than 0.5 from the correlations of measured values and distribution.

\section{Results}

On the analysis of the correlation of the values measured by SPAC-S LH and ARCHITECT LH, the values of subjects were classified into two groups. The values were distributed near the regression line represented by a solid line in most subjects (Fig. 1). However, the val- ues were distributed near the regression line represented by a broken line in about $10 \%$ of the subjects, and these subjects were those with a SPAC-S value lower than the actual value. We took more than 2 samples from these patients. Every measured value of these samples were almost the same and distributed near the same regression line. Thus, the ratio of measured LH values of SPAC-S LH and ARCHITECT LH (SPAC-S LH/ARCHITECT LH ratio) was calculated in individual samples, and the distribution was compared. The ratio ranged from 0.27 to 0.44 in 45 samples of 11 subjects, and 0.61-1.78 in the other 419 samples from 109 subjects. The distributions of SPAC-S LH/ ARCHITECT LH ratio in the 2 groups did not overlap. Thus, classification of two groups was easy when LH was assayed by SPAC-S LH and ARCHITECT LH. No sample was distributed between 0.45 and 0.6, and we defined 0.5 which was middle of the blank range as the discrimination ratio. The subjects with a SPAC-S LH/ARCHITECT LH ratio of lower than 0.5 were defined as patients with variant $\mathrm{LH}$. When a line, $\mathrm{y}=2 \mathrm{x}$ (dotted line), corresponding to a SPAC-S LH/ ARCHITECT LH ratio of 0.5, was drawn on the right (correlation in the subjects near the normal range) of Fig. 1, these 2 groups were clearly separated in the upper and lower regions of this line.

Almost all subjects with variant $\mathrm{LH}$, judged according to the definition determined above, were detectable in the correlation plot between SPAC-S LH and Centaur LH, but a few subjects with variant LH were not differentiated from normal LH (Fig. 2). On comparison of ARCHITECT LH and Centaur LH, the values measured by Centaur LH were slightly lower in the subjects with variant LH. When the subjects with variant $\mathrm{LH}$ were excluded, the correlation coefficient among the measurement systems was high (0.95-0.99). Based on the slopes of the regression lines the value measured by ARCHITECT LH was 1.2 times that measured by SPAC-S LH, and the value measured by Centaur LH was 1.1 times that measured by SPAC-S LH in the samples near the normal range.

The correlation coefficient among the measurement systems in FSH was high, 0.94-1.0 (Fig. 3). In samples near the normal range, the value measured by ARCHITECT FSH was 0.82 times that measured by SPAC-S FSH, and the value measured by Centaur FSH was 0.85 times that measured by SPAC-S FSH, based on the slopes of the regression lines.

As for ARCHITECT and Centaur, the LH and FSH 
Table 1. Measurement methods and standard

\begin{tabular}{ccccc}
\hline & IRMA (immunoradiometric assay) & & \multicolumn{2}{c}{ CLIA (chemiluminescence immunoassay) } \\
\cline { 2 - 3 } LH & SPAC-S & ARCHITECT & Centaur \\
FSH & 1st IRP-LH 68/40 & 2nd IS-LH 80/552 & 2nd IRP-LH 80/552 \\
PRL & 1st IRP-PRL 75/504 & & 2nd IS-FSH 78/549 & 2nd IS-FSH 94/632 \\
\hline
\end{tabular}

Table 2. Main analytical characterics as defined by manufactures of the three LH measurement systems

\begin{tabular}{|c|c|c|c|}
\hline & SPAC-S LH & ARCHITECT LH & Centaur LH \\
\hline Detection Limit (mIU/mL) & 0.1 & 0.07 & 0.07 \\
\hline Intra assay CV (\%) & 10 & 3.1 & 7.0 \\
\hline Inter assay $\mathrm{CV}(\%)$ & Data $(-)$ & 4.1 & Data $(-)$ \\
\hline \multirow[t]{3}{*}{ Cross reaction $(\%)$} & $\begin{array}{l}\text { TSH } \\
0.00\end{array}$ & $\begin{array}{c}\mathrm{TSH}(100 \mu \mathrm{IU} / \mathrm{mL}) \\
0.84\end{array}$ & $\begin{array}{c}\mathrm{TSH}(1,000 \mu \mathrm{IU} / \mathrm{mL}) \\
0.00\end{array}$ \\
\hline & $\begin{array}{l}\mathrm{hCG} \\
0.00\end{array}$ & $\begin{array}{c}\mathrm{hCG}(200,000 \mathrm{mIU} / \mathrm{mL}) \\
0.00\end{array}$ & $\begin{array}{c}\mathrm{hCG}(200,000 \mathrm{mIU} / \mathrm{mL}) \\
0.00\end{array}$ \\
\hline & $\begin{array}{l}\text { FSH } \\
0.00\end{array}$ & $\begin{array}{c}\text { FSH }(150 \mathrm{mIU} / \mathrm{mL}) \\
0.00\end{array}$ & $\begin{array}{c}\text { FSH }(150 \mathrm{mIU} / \mathrm{mL}) \\
0.00\end{array}$ \\
\hline
\end{tabular}

Table 3. Main analytical characterics as defined by manufactures of the three FSH measurement systems

\begin{tabular}{|c|c|c|c|}
\hline & SPAC-S FSH & ARCHITECT FSH & Centaur FSH \\
\hline Detection Limit (mIU/mL) & 0.1 & 0.05 & 0.3 \\
\hline Intra assay $\mathrm{CV}(\%)$ & 5.0 & 4.2 & 12.0 \\
\hline Inter assay $\mathrm{CV}(\%)$ & Data $(-)$ & 4.6 & Data $(-)$ \\
\hline \multirow[t]{5}{*}{ Cross reaction $(\%)$} & $\begin{array}{l}\text { TSH } \\
0.00\end{array}$ & $\begin{array}{c}\mathrm{TSH}(100 \mu \mathrm{IU} / \mathrm{mL}) \\
0.00\end{array}$ & $\begin{array}{c}\mathrm{TSH}(1,000 \mu \mathrm{IU} / \mathrm{mL}) \\
0.00\end{array}$ \\
\hline & hCG & $\mathrm{hCG}(200,000 \mathrm{mIU} / \mathrm{mL})$ & hCG $(200,000 \mathrm{mIU} / \mathrm{mL})$ \\
\hline & 0.00 & 0.00 & 0.00 \\
\hline & LH & LH (250 mIU/mL) & LH (300 mIU/mL) \\
\hline & 0.00 & 0.00 & 0.00 \\
\hline
\end{tabular}

Table 4. Main analytical characterics as defined by manufactures of the three PRL measurement systems

\begin{tabular}{lccc}
\hline & SPAC-S PRL & ARCHITECT PRL & Centaur PRL \\
\hline Detection Limit (ng/mL) & 0.3 & 0.6 & 0.3 \\
Intra assay CV (\%) & 7.0 & 3.8 & 5.0 \\
Inter assay CV (\%) & Data $(-)$ & 4.7 & Data $(-)$ \\
Cross reaction (\%) & TSH & TSH $(20,000 \mu \mathrm{IU} / \mathrm{mL})$ & TSH $(1,000 \mu$ IU $/ \mathrm{mL})$ \\
& 0.00 & hCG $(100,000 \mathrm{mIU} / \mathrm{mL})$ & 0.00 \\
& hCG & 0.00 & hCG $(200,000 \mathrm{mIU} / \mathrm{mL})$ \\
& 0.00 & LH $(5,000 \mathrm{mIU} / \mathrm{mL})$ & 0.00 \\
& LH & 0.00 & $\mathrm{LH}(250 \mathrm{mIU} / \mathrm{mL})$ \\
& 0.00 & FSH $(1,000 \mathrm{mIU} / \mathrm{mL})$ & 0.00 \\
& FSH & 0.00 & $\mathrm{LH}(200 \mathrm{mIU} / \mathrm{mL})$ \\
\end{tabular}



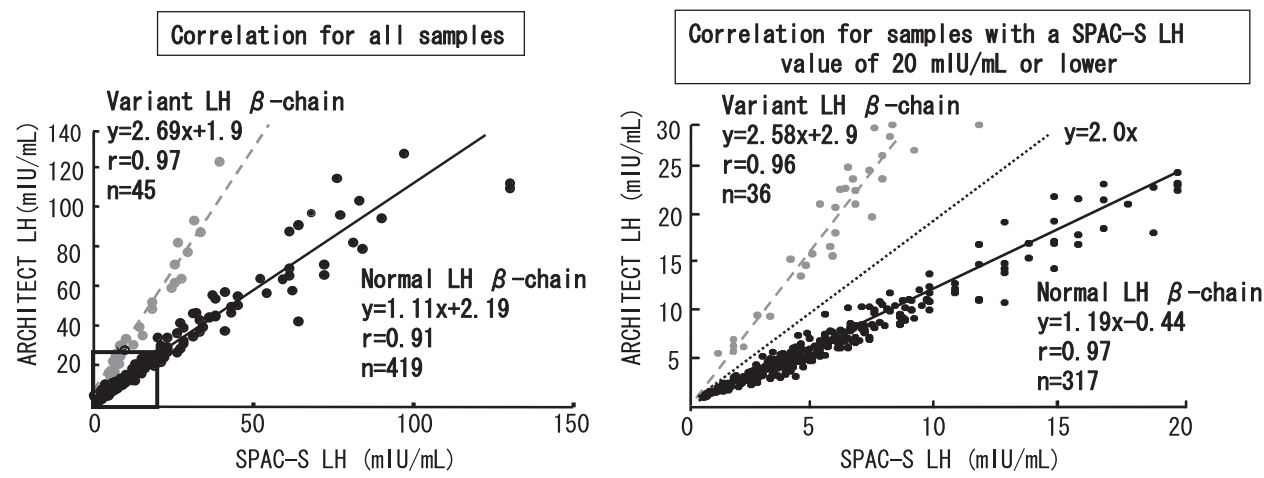

SPAC-S LH/ARCHITECT LH ratio
Variant LH: $0.34 \pm 0.05(0.27 \sim 0.44)$
Normal LH: $0.93 \pm 0.15(0.61 \sim 1.78)$
mean \pm standard deviation (lowest value $\sim$ highest value)

Judgement criterion for variant LH SPAC-S LH/ARCHITECT LH ratio $<0.5$

Fig. 1. Judgment of variant LH
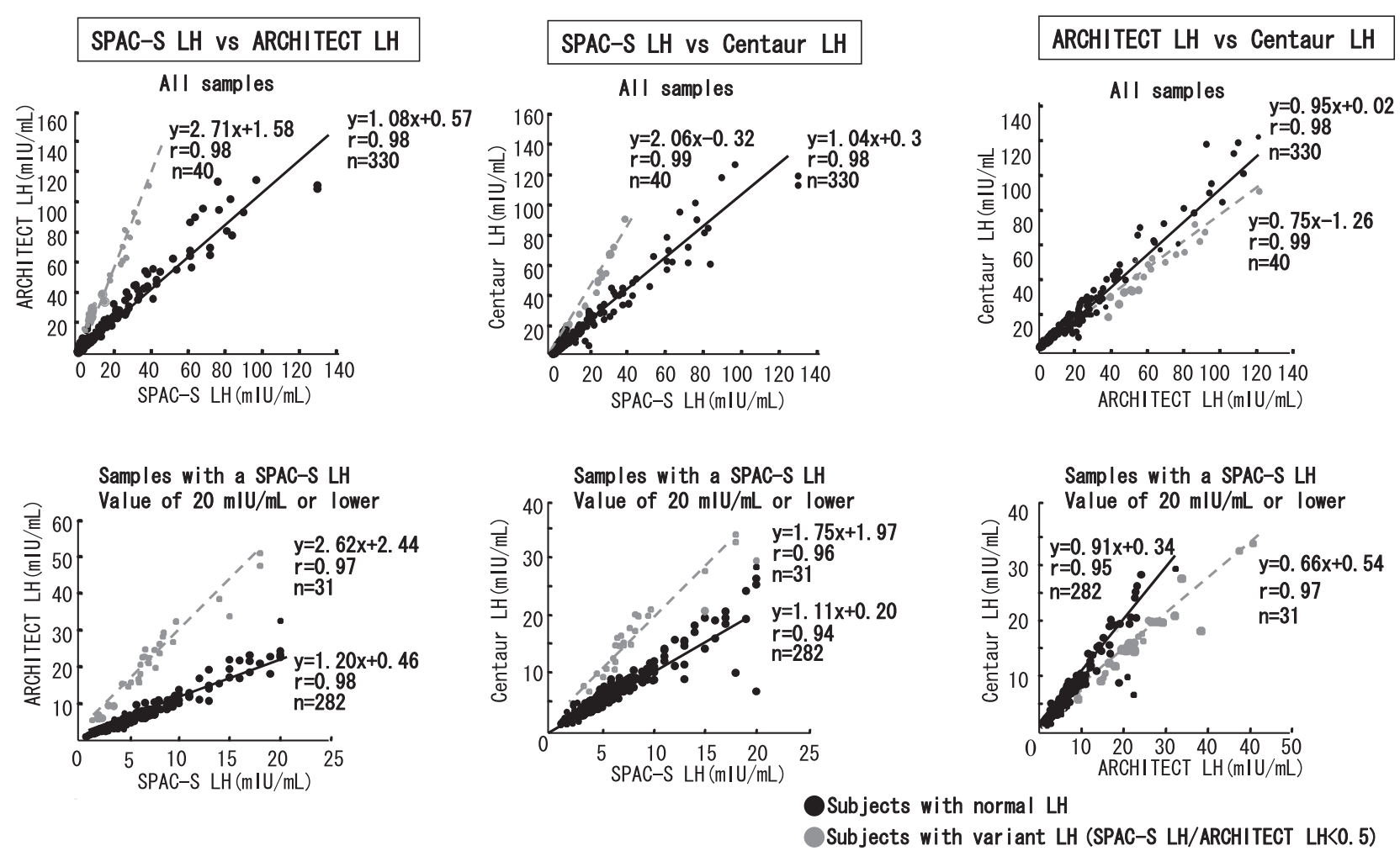

Fig. 2. Correlation of values among the measurement systems (LH)

values were close to those measured by SPAC-S, but the LH/FSH ratio measured by ARCHITECT was 1.25 times that measured by SPAC-S, and the ratio measured by Centaur was 1.37 times that measured by SPAC-S, based on the slopes of the regression lines (Fig. 4).

Seven patients were diagnosed with normoestrogen- ic amenorrhea based on the LH and FSH values measured by SPAC-S, and 3 of these had variant LH based on the criteria described above. GnRH tests were performed in these 3 patients with variant $\mathrm{LH}$ and 1 subject with normal LH (Fig. 5). Excess responses of LH were observed in 2 of the patients with variant $\mathrm{LH}$, suggesting PCOS. In these 2 patients, PCOS could be 

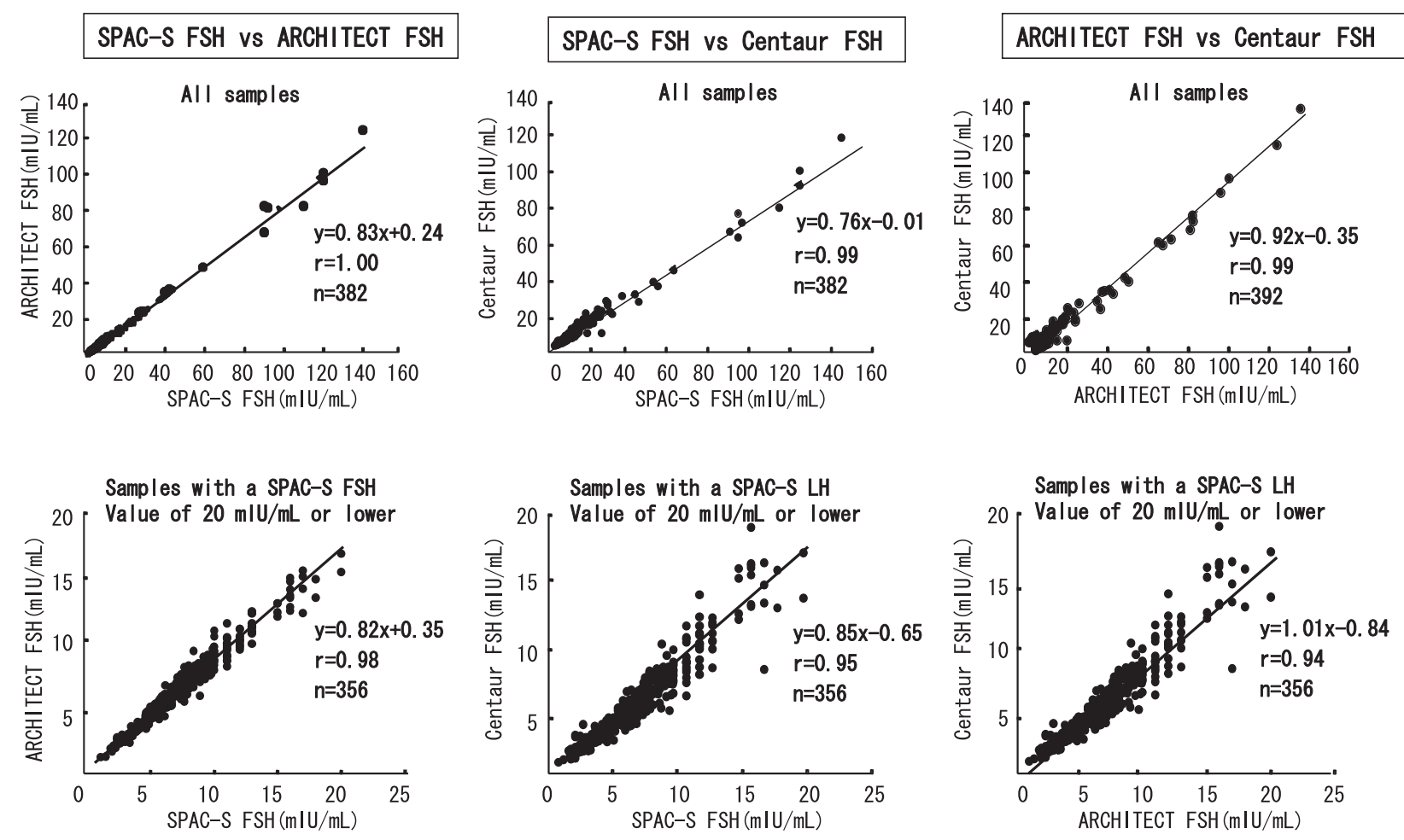

Fig. 3. Correlation of values among the measurement systems (FSH)
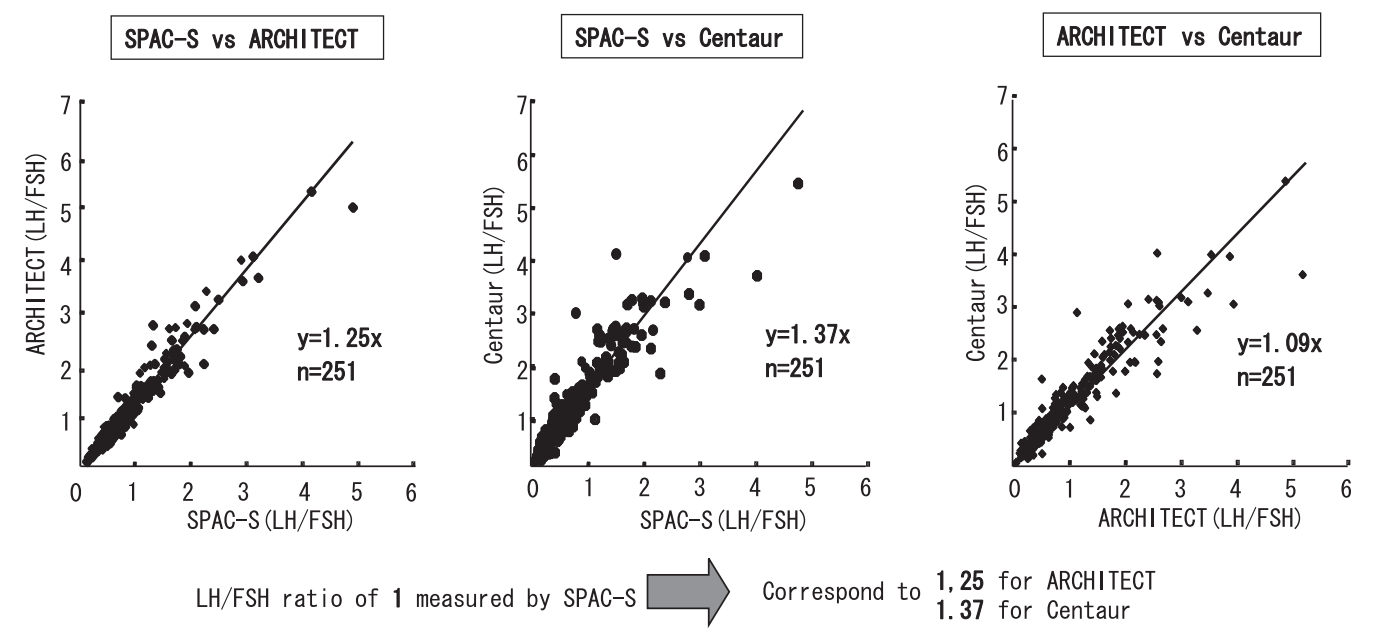

Fig. 4. Correlation of LH/FSH among the measurement systems (samples with a SPAC-S LH value of $20 \mathrm{mIU} / \mathrm{mL}$ or lower excluding subjects with variant $\mathrm{LH}$ )

diagnosed by ARCHITECT LH and Centaur LH, because the LH values measured by these systems were already high before the GnRH injection.

The correlation coefficient of PRL among the measurement systems was $0.96-0.99$ (Fig. 6). The values measured by ARCHITECT PRL was 1.92 times that measured by SPAC-S PRL, and the value measured by
Centaur PRL was 1.64 times that measured by SPAC-S, based on the slope of the regression lines, showing large differences.

Changes in the measured values within the menstrual cycle were similar among the measurement systems (Fig. 7).

LH and FSH were low from the early gestational pe- 

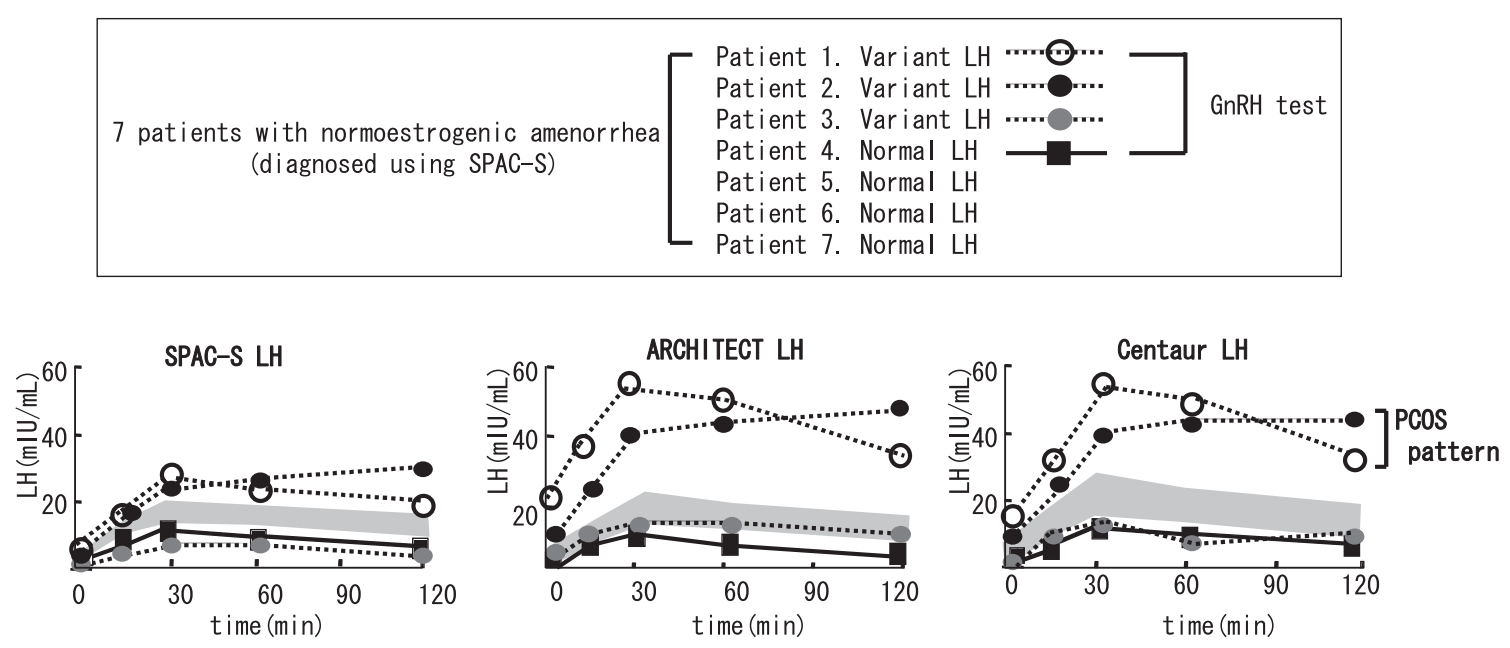

Fig. 5. GnRH test in patients diagnosed with normoestrogenic Amenorrhea (diagnosed using SPAC-S)
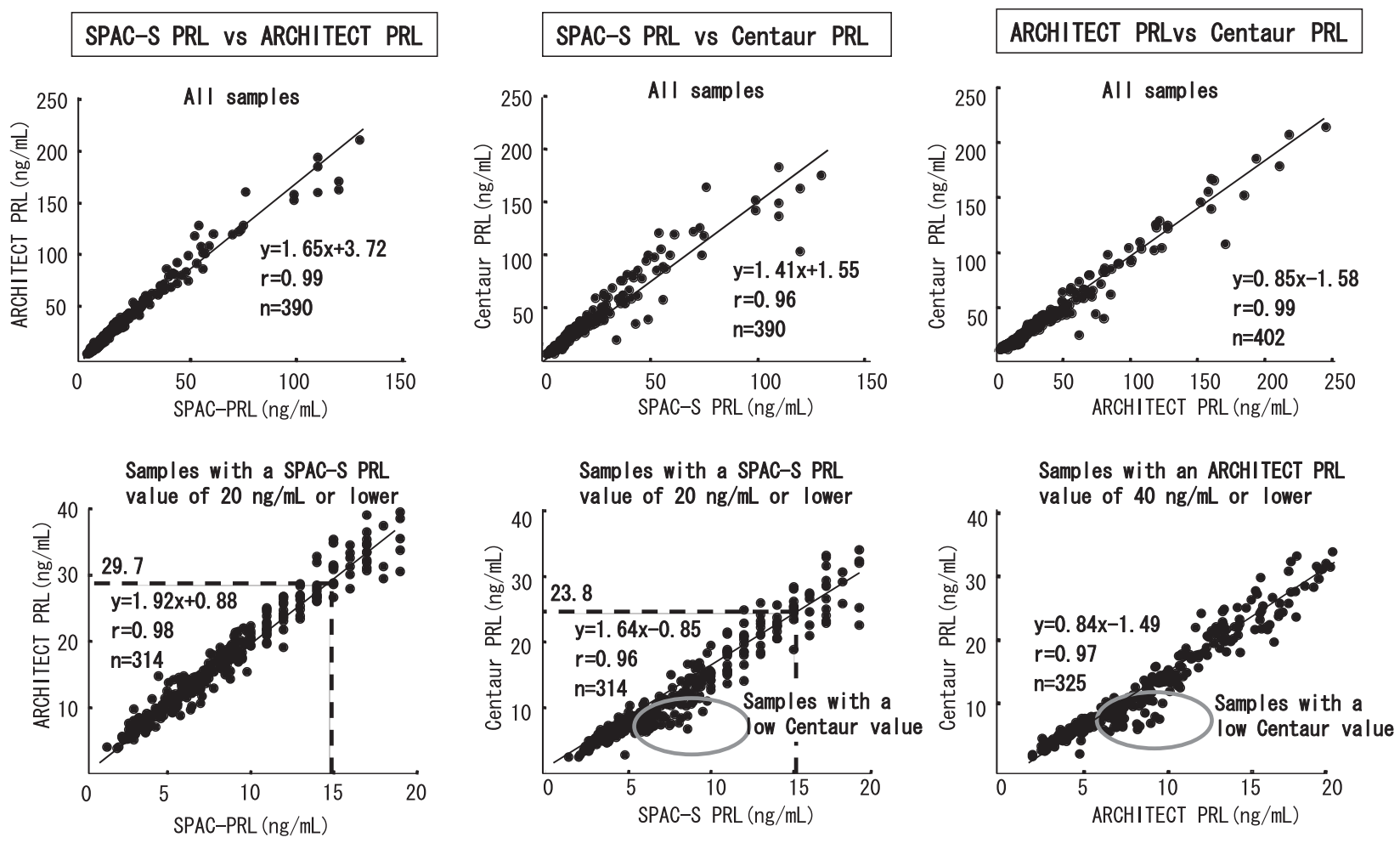

Fig. 6. Correlation of values among the measurement systems (PRL)

riod in all measurement systems, suggesting that crossreactivity with hCG was almost absent (Fig. 8). Values of PRL were high through the early to the late gestational periods.

Among the ovulatory disorders, the LH and FSH values measured by SPAC-S were largely deviated compared to those measured by ARCHITECT and Centaur in PCOS. The PRL values measured by SPAC-S also deviated from other 2 systems in hyperprolactinemia (Fig. 9). 


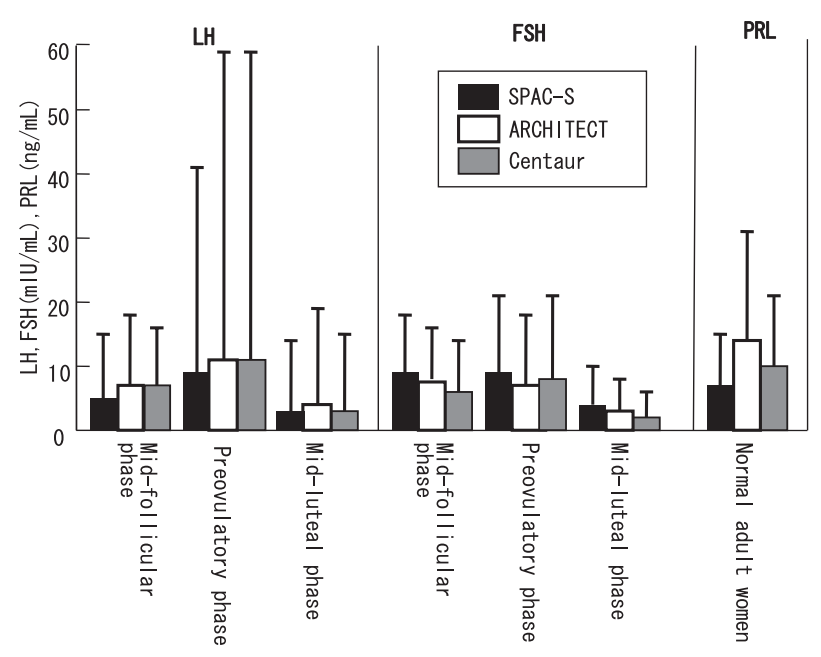

Fig. 7. Measurement values in women with a normal menstrual cycle (Mean value $+2 \mathrm{SD}$ after logarithmic conversion)

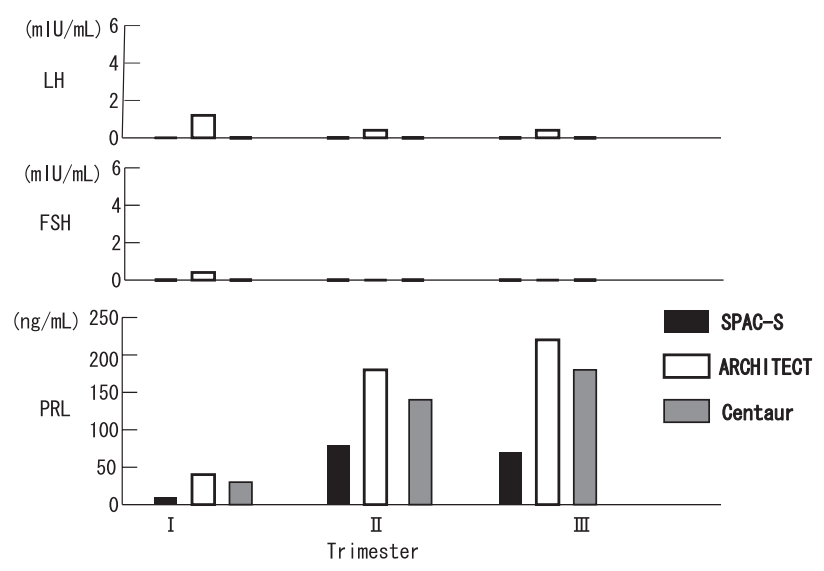

Fig. 8. Measured values in the gestational periods

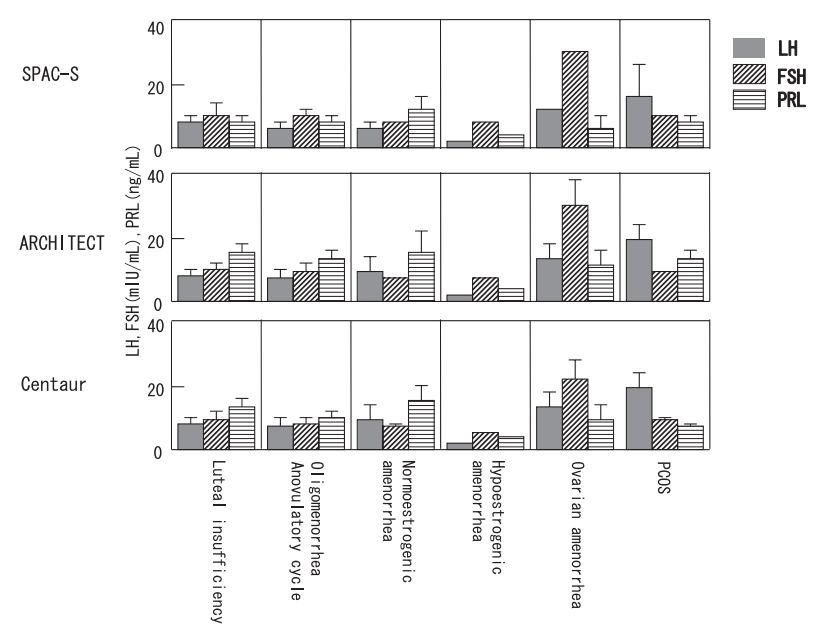

Fig. 9. Measured values in patients with various disorders (Standard value $+\mathrm{SE})$

\section{Discussion}

Recently the main measurement systems of LH, FSH, and PRL have shifted from RIA methods to nonRIA ones. The measurement systems investigated in this study are widely used in Japan, and good results have been obtained in basic studies [2-10]. SPAC-S uses the IRMA method, and ARCHITECT and Centaur use the CLIA method (non-RIA). Since different standards are used in some systems, we anticipated difficulty in the comparison of measured values among the several measurement systems. Thus, clinical problems were investigated with regard to correlation of the values measured by the measurement systems and the standards.

In patients with variant $\mathrm{LH}$, a mutation is observed in the gene sequence of LH $\beta$-chain, and the biological activity is higher than that of the wild type in vitro, while its half-life is short [11]. SPAC-S LH system uses two different monoclonal antibodies that react with $\beta$ subunit of $\mathrm{LH}$ and the intact $\mathrm{LH}$ dimer. The most popular mutation of LH exists just around the reaction point of monoclonal antibody used in SPACS LH. Values of LH assayed by SPAC-S LH in cases with variant $\mathrm{LH}$ were lower than actual values. ARCHITECT LH and Centaur LH systems recognized both variant and wild type $\mathrm{LH}$. We can thus detect variant $\mathrm{LH}$ using the ratio of measured values of SPAC-S and other systems. Takahashi et al. studied prevalence of variant LH in Japanese women using SPAC-S LH and Immulyze LH (Nippon DPC Corporation, Chiba, Japan), and reported that $8.5 \%$ of Japanese female have variant LH [12]. Furthermore, Takahashi et al. confirmed that variant LH diagnosed by deviation of measured values between the two assay systems was proper using DNA analysis $[12,13]$. They reported that gene mutations of $\operatorname{Trp}^{8}$ to $\mathrm{Arg}^{8}$ and $\mathrm{Ile}^{15}$ to $\mathrm{Thr}^{15}$ were seen in variant LH cases [12]. The rationale of diagnosis we used for diagnosis of variant LH was the same as that of previous reports. We used deviation of measured value between two assay systems including SPAC-S LH. Our combination of SPAC-S LH and ARCHITECT LH seemed better than that of previous reports because of its superior discrimination ability. Diagnosis of variant LH in this report would be proper although we did not confirm gene mutation. In this study, subjects with SPAC-S LH/ARCHITECT LH ratio lower than 0.5 were differentiated as having variant LH, accounting for $10.7 \%$ of all subjects. This finding 
was not contradictory to the study reporting that about $8.5 \%$ of Japanese females have variant LH [12]. When variant LH was judged using SPAC-S LH/Centaur LH ratio, clear differentiation was not possible in some subjects. In addition, the regression line for the patients with variant $\mathrm{LH}$ was different from that for the subjects with normal LH in the correlation plot between the Centaur LH and ARCHITECT LH values, suggesting that Centaur LH might not be completely capable of detecting variant LH. ARCHITECT LH is most accurate for the measurement of $\mathrm{LH}$ in patients with variant $\mathrm{LH}$.

The correlations among the values measured by the 3 systems were high for LH, FSH, and PRL. The LH and FSH values alone were relatively close among the 3 systems, but the LH/FSH ratio measured by SPAC-S was markedly different from those measured by the other 2 systems. A high level of LH alone is considered important as an essential item of the diagnostic criteria for PCOS established by the Japan Society of Obstetrics and Gynecology [1]. The judgment criterion of a high level of LH relative to FSH has usually been set to LH/FSH ratio $>1.0$ when SPAC-S is used. This criterion would be LH/FSH ratio $>1.25$ for ARCHITECT, and LH/FSH ratio $>1.4$ for Centaur based on correlations of LH/FSH ratio among the 3 kits. When we make a diagnosis of PCOS, we should be aware which measurement system is used in measurement of LH. As for measured PRL values, the value measured by ARCHITECT PRL was 1.92 times higher and the value measured by Centaur PRL was 1.64 times higher than that measured by SPAC-S PRL, showing greater differences among the 3 kits. A SPAC-
S PRL value of 15 or higher has been used as a diagnostic criterion of hyperprolactinemia. The corresponding values measured by ARCHITECT PRL and Centaur PRL are 29.7 or higher and 23.8 or higher, respectively, and thus, caution is necessary when making the diagnosis of hyperprolactinemia based on there kits.

In the patients with concomitant PCOS and variant $\mathrm{LH}$, the values of LH measured by SPAC-S might not meet the criterion for high $\mathrm{LH}$, suggesting that the patients may be mistaken for hypothalamic normoestrogenic anovulation. When PCOS is suspected clinically but the LH value measured by SPAC-S is not high, it is necessary for the diagnosis to perform additional measurements of LH and FSH using other systems or a GnRH test to examine the PCOS pattern.

Based on the above findings, information of the standard values of measurement system is important for the assessment of LH, FSH, and PRL, and particularly, we should be careful in the diagnoses of PCOS and hyperprolactinemia. Particularly, for the judgment criteria of high LH in the diagnosis of PCOS, LH/FSH ratio $>1$ measured by SPAC-S, $>1.25$ measured by ARCHITECT, and $>1.37$ measured by Centaur may be appropriate. In addition, it is necessary to make the diagnostic criteria for the disorders corresponding to each measurement system or to make converter index for common value to all measurement systems.

\section{Acknowledgements}

We thank Abbott Japan Inc. and Bayer Medical Co., Ltd. for the measurements of samples.

\section{References}

1. Sugimoto O, Aono T (1993) The Committee for Reproductive and Endocrine in Japan Society of Obstetrics and Gynecology. Annual report (1991-1992) for the determination of diagnostic criteria for polycystic ovary syndrome. Acta Obstet Gynaecol Jpn 45: 1359-1367 (In Japanese).

2. Yasui T,Ueda T, Shitukawa K, Kanematsu T, Azuma K, Irahara M, Aono T (1988) Basic and clinical investigation of LH/FSH-IRMA system using pituitary gonadotropin standard. In: Aono T (ed) Progression of LH/FSH measurement method. LH/FSH Research Group, Tokyo: 35-46 (In Japanese).

3. Yasui $\mathrm{T}$, Ikawa $\mathrm{H}$, Saito $\mathrm{S}$, Matsuzaki $\mathrm{T}$, Ueda $\mathrm{T}$,
Shitsukawa K, Kanematsu T, Azuma K, Irahara M, Aono (1989) Basic and clinical investigation of PRLIRMA system using WHO standard. In: Aono T (ed) Progression of Prolactin Measurement Method. PRL Research Group, Tokyo: 26-34 (In Japanese).

4. Irahara M, Yasui T, Azuma K, Aono T (1988) Fundamental studies on immunoradiometric assays (SPACS LH, SPAC-S FSH) for measurement of serum LH and FSH using the pituitary gonadotropin standard. Horumon to Rinsho 36: 1223-1230 (In Japanese).

5. Aono T, Kumamoto Y, Sasaki Y, Tamada T (1989) Multiple basic and clinical studies on immunoradiometric assay (SPAC-S Prolactin Kit) for measurement 
of serum prolactin using WHO standard sample. Horumon to Rinsho 37: 87-101 (In Japanese).

6. Miura N, Imaizumi K, Sakai S, Kondo F, Ogitsu N, Ezaki K (2000) Determination of fertility hormone's reference ranges in ARCHITECT i2000 system. Jpn J Clin Lab Automat 25: 213-215 (In Japanese).

7. Minakata R, Sato R, Terada M, Katayama R, Sugiyama $\mathrm{T}$ (1999) Investigation of blood E2, LH, FSH, progesterone, and prolactin measurements using ARCHITECT analyzer i2000 (DAINABOT Co.). Rinsho Kensakiki Shiyaku 22: 429-438 (In Japanese).

8. Umezu M, Kitano M, Yamazaki Y, Arakawa S, Arakawa Y, Shimizu T (1999) Measurement of fertility hormones by ARCHITECT $i$ System in comparison to other assay systems. Horumon to Rinsho 47: 87-94 (In Japanese).

9. Kishino Y, Morishima Y, Akiyama T, Ooba Y, Ishikawa M, Hoshiai H (1996) Fundamental evaluation of determination of LH, FSH and PRL in serum by ACS 180 .
Horumon to Rinsho 44: 89-96 (In Japanese).

10. Nakamura S, Miura A, Konno T, Itoh C, Shirayanagi Y (1998) Fundamental evaluation of measurement of LH, FSH, PRL, E2, P4, testosterone using automatic chemiluminescence immunoassay "Chemilumi ACS: Centaur”. Igaku to Yakugaku 40: 541-545 (In Japanese).

11. Lamminen T, Huhtaniemi I (2001) A common genetic variant of luteinizing hormone; relation to normal and aberrant pituitary-gonadal function. Eur $J$ Pharmacol 414: $1-7$.

12. Takahashi K, Ozaki T, Okada M, Kurioka H, Kanasaki $\mathrm{H}$, Miyazaki (1999) Increased prevalence of luteinizing hormone $\beta$-subunit variant in patient with premature ovarian failure. Fertil Steril 71: 96-101.

13. Takahashi K, Ozaki T, Kanasaki H, Miyazaki K (2001) Successful pregnancy in a woman with ovarian failure associated with mutation in the $\beta$-subunit of luteinizing hormone. Horm Res 55: 258-263. 